\title{
The Search for Brown Dwarfs around White Dwarfs
}

\author{
J. Farihi, E. E. Becklin, B. Zuckerman \\ University of California Los Angeles, 8371 Mathematical Sciences \\ Building, Los Angeles CA 90095-1562
}

\begin{abstract}
The infrared search for substellar companions to nearby white dwarfs has been going on for a little more than a decade. The most recent phase has been a wide field proper motion search carried out primarily at Steward Observatory, where we are complete down to $J=18$. Earlier phases included near field searches at the IRTF and Keck Observatory. In the last year we have discovered ten previously unrecognized faint proper motion companions. Of the recent discoveries, most are white dwarfs and a few $M$ dwarfs. GD165B, discovered in 1988 as part of our program, is still the only known companion to a white dwarf with spectral type later than $\mathrm{M}$.
\end{abstract}

\section{Description of the Current Survey}

We are conducting a proper motion survey at $1.25 \mu \mathrm{m}$ for low mass stellar and substellar companions to nearby white dwarfs. The advantage of such a search is that white dwarfs are faint in the infrared, enabling discovery of brown dwarf companions even relatively close to the primary (Zuckerman \& Becklin 1987 \& 1992; Becklin \& Zuckerman 1988). The current wide field survey has been conducted mainly at the Steward Observatory beginning in 1991 . There on the $2.3 \mathrm{~m}$ Bok telescope we have used a NICMOS array and a camera developed by Rieke \& Rieke (Rieke et al. 1993). With a 3 square arcminute field of view, we can detect faint companions $2-90^{\prime \prime}$ from the primary white dwarf. We acquire five dithered 90 second images at $J$ band which enables detection of objects as faint as $J=19$ in the best observing conditions.

Generally we acquire two epochs for each field surrounding the primary white dwarf. The typical white dwarf in our sample has a small known proper motion around $0.1-0.2^{\prime \prime} / \mathrm{yr}$. Since the mid 1990's, the white dwarfs will have moved $\sim 0.5-1.0^{\prime \prime}$ relative to background stars and galaxies. We have found that when there are more than 5 objects which appear in both epoch fields, the scatter in their measured positions is approximately $0.2^{\prime \prime}$. Thus, we can easily detect a $0.5^{\prime \prime}$ displacement of the primary relative to background objects in the field. Hence with a baseline $\geq 5$ yrs between epochs, companions that move with the primary will stand out well against background objects.

The white dwarfs chosen for our survey come from the catalog of McCook \& Sion (1999). Our targets consist mainly of white dwarfs with modest known proper motions selected from the Lowell \& Luyten surveys along with some objects from the Palomar-Green survey. The reason for choosing white dwarfs 
with smaller proper motions is that stars with smaller U,V space velocities are statistically more likely to be members of the young disk (Eggen 1996) and brown dwarfs are brightest when they are young. White dwarfs are evolved but not necessarily old; a $3 M_{\odot}$ A0 star can evolve into a white dwarf in only 0.6 Gyr. Hence by selecting slow movers there is a greater likelihood that we are looking around younger white dwarfs. The distance to a typical white dwarf in our sample of $\sim 240$ targets is around $50 \mathrm{pc}$.

\section{Discoveries}

GD165B was discovered in the very first incarnation of the search for brown dwarfs around white dwarfs conducted by Becklin \& Zuckerman (1988). It still remains the only known possible brown dwarf companion to a white dwarf and the only known companion to a white dwarf with spectral type later than M. Zuckerman \& Becklin (1992) discovered or measured over $20 \mathrm{M}$ dwarf companions to white dwarfs in near field searches conducted at the IRTF and UKIRT of about 200 white dwarf primaries.

In the current wide field survey, approximately 100 of our white dwarfs have been analyzed and we have found 10 previously unrecognized proper motion companions to their respective primaries. Hence at present we are finding about $10 \%$ of our sample have wide companions. Three of these companions are likely $\mathrm{M}$ dwarfs and the other seven are either confirmed or likely white dwarfs. It appears that none of these companions are substellar candidates. Thus we conclude that not only is there a brown dwarf desert around white dwarfs at close separations but our data analyzed so far indicates that this trend likely persists at wider separations as well.

\section{Implications for Star/Brown Dwarf Formation}

One possible explanation for the apparent deficit of substellar companions to white dwarfs is that binary star formation may favor equally massive components. There exist models which favor two accreting protostellar components in a binary system equilibrating in mass as they form. One model even predicts that unequal mass binaries are more likely to form at wide separations due to accretion issues (Bate 2000). Yet there are many known WD/RD pairs at close as well as wide separations (Zuckerman \& Becklin 1992; Silvestri et al. 2001). These systems would have had very unequal mass components when on the zero age main sequence. Radial velocity searches indicate that brown dwarf companions to main sequence stars are rare at separations of less than 5 AU. Results from 2MASS and other studies indicate that brown dwarf companions may be more common at wide separations (Gizis et al. 2001).

Companions to white dwarfs suffered a change in their orbital separations since the time when the primary was on the main sequence. For companions separated by less than a few AU when the host star ascends the AGB, common envelope interaction will cause the orbit to decay into an even closer system. For companions outward of $3 \mathrm{AU}$, the orbit will expand by a factor of $M_{\mathrm{ms}} / M_{\mathrm{wd}}$ (Jeans 1924). Thus one can expect wide binary systems with one white dwarf component to have separations that are roughly 2 to 7 times larger than when 
both components were on the main sequence. A system with a white dwarf and a widely separated brown dwarf companion may be vulnerable to disintegration through close encounters with nearby molecular clouds or other stellar systems over a few billion years due to the low mass nature of brown dwarfs.

Because brown dwarfs continue to grow dimmer as they age, we must consider the limits of our sensitivity. The ages of white dwarfs are still being refined and the refinements tend to make them older than previous models predicted. For example, the difference in predicted cooling times of a typical mass white dwarf with a pure carbon core versus a pure oxygen core can be as much as 2 Gyr. What this means for our search is that it is possible that the ages of white dwarfs could be pushing brown dwarf luminosities past our sensitivity limits into the late $\mathrm{T}$ dwarf regime and beyond. Based on their space motions and model predicted cooling times however, the total age of a typical white dwarf in our sample is $1-3 \mathrm{Gyr}$, assuming average masses. Hence if the models are correct, we don't expect the age of our sample to play a factor in detecting brown dwarfs down to about $30 M_{\mathrm{J}}$ for the youngest white dwarfs in our sample.

\section{Conclusions}

The search for brown dwarfs around white dwarfs is of continuing interest for several reasons. One is that we do not yet know which temperature / spectral class is the lower cut off for a minimum mass star. Until we start to discover and study more companions which straddle and cross the stellar / substellar boundary, the model predicted minimum temperature value is the only way we can judge whether an object is a brown dwarf or a low mass star if an accurate age is not known. Second, by searching for M, L, and T-type companions to white dwarfs, we are probing ages when brown dwarfs have cooled significantly and low mass stars of course have not. When our search or future searches begin to find low temperature (L-type) companions around old stars such as some white dwarfs, we can then better constrain stellar / substellar cooling model temperatures, ages and masses. Also by conducting this kind of search we want to shed light on the formation of companions to intermediate mass stars, the progenitors of the white dwarfs we see today.

\section{References}

Bate, M. R. 2000, MNRAS, 314, 33

Becklin, E. E., \& Zuckerman, B. 1988, Nature, 336, 656

Eggen, O. J., 1996, AJ111, 466

Gizis, J. E., Kirkpatrick, J. D., Burgasser, A., Reid, I. N., Monet, D. G., Liebert, J., \& Wilson, J. C. 2001, ApJ, 551, L163

Jeans, J. H. 1924. MNRAS, 85, 2

McCook, G. P., \& Sion, E. M. 1999, ApJS, 121, 1

Rieke, M. J., Rieke, G. H., Green, E. M., Montgomery, E. F., \& Thompson, C. L. 1993, SPIE, 1946, 179 
Silvestri, N. M., Oswalt, T. D., Wood, M. A., Smith, J. A., Reid, I. N., Sion, Edward M. 2001, AJ, 121, 503

Zuckerman, B., \& Becklin, E. E. 1987, ApJ, 319, L99

Zuckerman, B., \& Becklin, E. E. 1992, ApJ, 386, 260

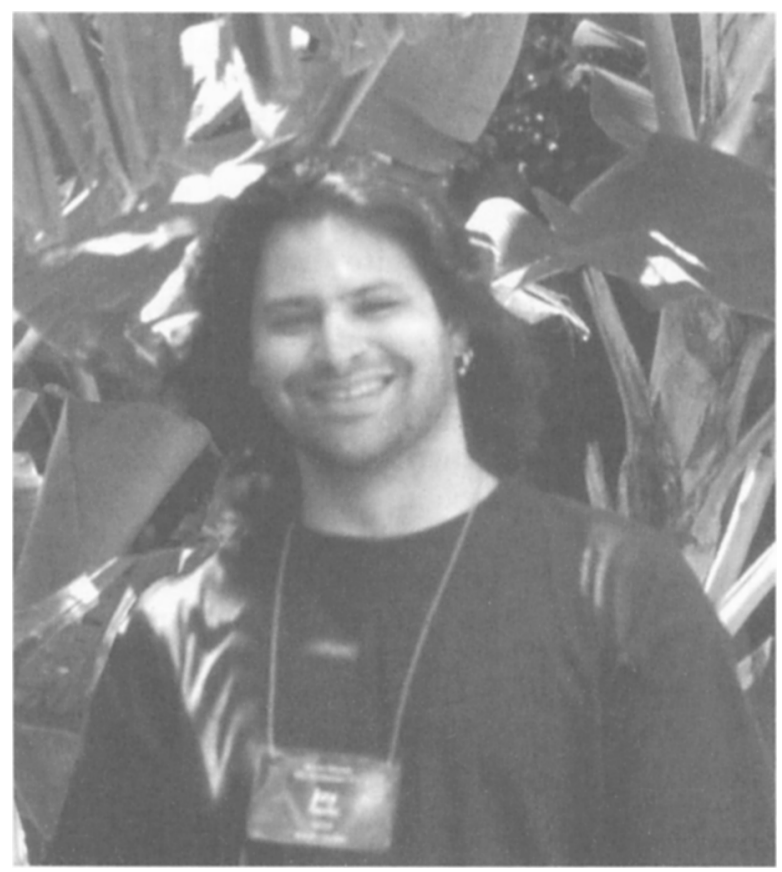

Jay Farihi 